\title{
Salt restriction and the voluntary intake of ethanol in rats
}

\author{
L. A. GRUPP \\ University of Toronto and Addiction Research Foundation, Toronto, Ontario, Canada
}

and

\author{
R. B. STEWART and E. PERLANSKI \\ University of Toronto, Toronto, Ontario, Canada
}

\begin{abstract}
To investigate the effect of salt restriction on the voluntary intake of ethanol, three groups of rats were offered a choice between ethanol $(3 \%, 6 \%$, or $12 \% \mathrm{v} / \mathrm{v})$ and water every 3rd day with only ethanol available on the two intervening "forced" days. A fourth group was offered only water throughout. Animals fed a nutritionally adequate diet preferred ethanol in concentrations below $6 \%$ (Phase 1) and continued to drink similar amounts of ethanol in Phase 2 when fed only a low-sodium diet. When a brief series of four injections of the salt-losing diuretic furosemide was added to the low-sodium regimen (Phase 3), most rats stopped preferring ethanol and decreased the amount of ethanol they consumed. Upon return to the nutritionally adequate diet (Phase 4), the consumption of ethanol returned to previous levels. Forced-day consumption of ethanol was unaffected by these treatments. Control groups of animals that were offered similar concentrations of ethanol and maintained on the nutritionally adequate diet throughout, but given the diuretic, did not decrease their ethanol consumption. These findings suggest that salt restriction in combination with a diuretic-natriuretic is an effective means to reduce voluntary ethanol intake, and suggest that sodium and/or some aspect of the mechanisms regulating sodium balance may modulate ethanol preference and intake.
\end{abstract}

When insufficient amounts of sodium are available to maintain sodium balance or repair a sodium deficit, animals will respond endocrinologically by secreting aldosterone to prevent sodium loss through the urine and behaviorally by seeking out and consuming substances that contain significant amounts of sodium. The latter reflects what has been termed a craving for salt and can be observed both in animals who travel hundreds of miles to reach salt licks (Denton, 1967) and in humans, where patients with adrenal insufficiency (Richter, 1942) or tumor (Wilkins \& Richter, 1940) keep themselves alive by consuming sodium-rich substances.

The idea that such a sodium hunger, on the one hand, and the appetite for alcohol, on the other, might be related stems from a number of observations. Iida (1957) was the first to demonstrate that mice would increase their intake of an otherwise rejected solution of alcohol (15\%) if the alcohol was offered in a $1 \%$ saline solution, or if the animals were pretreated with daily intraperitoneal injections of saline or desoxycorticosterone acetate

The authors gratefully acknowledge the assistance of Hau Lei and Henzel Jupiter of Computer Services, Addiction Research Foundation, for the statistical analysis and of Val Cabral for preparing the typescript. Furosemide was generously provided by Dieter Baun of Novopharm Ltd., Toronto, Canada. This research was supported by the Addiction Research Foundation. The authors' mailing address is: Department of Pharmacology, University of Toronto, Toronto, Canada M5S 1A8.
(DOCA), a mineralocorticoid that causes sodium retention. Similarly, Linkola (1982) and Linkola, Fyhrquist, and Forsander (1977) have shown that rats selected by outbreeding to drink large amounts of alcohol (AA strain) showed both a higher level of sodium retention and a higher concentration of the endogenous mineralocorticoid aldosterone than did another strain (ANA) selected not to drink alcohol. Finally, recent work from our laboratory has shown that a high salt diet can enhance ethanol intake and reduce intoxication, whereas a DOCA-induced salt appetite will reduce ethanol intake and enhance intoxication (Grupp, Elias, Perlanski, \& Stewart, in press; Grupp, Perlanski, \& Stewart, 1984).

Clinical evidence also points to a link between the regulation of sodium balance and alcohol use. Although there is some controversy surrounding the mechanism, there is a great deal of documented evidence to show that patients with Laennec's (alcoholic) cirrhosis and in an advanced stage of liver disease show marked elevations in both plasma and urine levels of renin and aldosterone (Epstein, Sancho, \& Haber, 1979). Addison's disease is one in which an aldosterone deficiency, due to adrenocortical hypofunction, leads to low blood sodium concentrations. If uncorrected, an Addisonian crisis results. This crisis has been observed to be similar both clinically and biochemically to delirium tremens, one of the more severe symptoms of alcohol withdrawal (Smith, 1950). Furthermore, Wood- 
bury, Timiras, and Vernadakis (1957) have shown that an increase in intracellular brain sodium concentration, as would occur with adrenocortical hypofunction, results in an increased brain excitability. It is this latter condition that is highly correlated with acute alcohol withdrawal. Finally, an association between hypertension and high sodium intake has been proposed (Dahl, 1977), as has the association between hypertension and high alcohol intake (Ashley \& Rankin, 1979; Rubin, 1982). It is possible that both these agents produce the hypertensive condition by different mechanisms. However, at the present stage of our knowledge, it is also possible that some of the causes of hypertension (e.g., sodium intake) may also predispose to alcohol use, and vice versa (Mathews, 1979).

On the strength of these observations, the present experiment examines one aspect of the possible relationship between sodium and alcohol intake by investigating whether sodium restriction either alone or in combination with a salt-losing diuresis would alter the intake of ethanol in rats.

\section{METHOD}

\section{Subjects}

The subjects were 48 naive female Wistar rats weighing $220-270 \mathrm{~g}$ at the beginning of the experiment. All animals were housed individually in cages equipped with a glass feeder cup and two Richter tubes placed $5 \mathrm{~cm}$ apart. A 12-h light-dark cycle was in effect throughout, with lights on at $7 \mathrm{a} . \mathrm{m}$.

\section{Procedure}

The animals were distributed according to weight into four groups of 12 animals, each allowed continuous access to either distilled water or $3 \%, 6 \%$, or $12 \%$ ethanol $(v / v)$ prepared in distilled water. The experiment was run in four phases, each comprising 6-13 3-day cycles. Each cycle consisted of two consecutive 24-h periods, during which each animal was offered only its respective ethanol solution to drink (forced days), followed by a third 24-h period, during which both ethanol and water were available (choice day). The positions of the water and ethanol tubes were alternated regularly. Progression from one phase to the next was done only after consumption had stabilized.

Phase 1. All animals were fed a nutritionally adequate purified control (C) diet (No. 5755, Ralston Purina Co.) for a period of 18 days (6 cycles). This diet contained $0.29 \%$ sodium by assay.

Phase 2. Half the animals from each of the four groups were switched to a low sodium (LS) diet equivalent in every respect to the control diet except for the sodium content $(0.0079 \%$ sodium by assay, No. 5881 , Ralston Purina Co.). The other half of the animals continued on the control diet. The animals were subdivided such that in each case the mean weights of the two diet groups consuming the same ethanol solution were equal. This phase lasted 39 days ( 13 cycles).

Phase 3. All animals continued to consume their respective ethanol solutions and diets. However, on both forced days of the first and of the fourth cycles only, all animals were given an ip injection of the diuretic furosemide at a dose of $60 \mathrm{mg} / \mathrm{kg}$. This phase lasted 30 days ( 10 cycles).

Phase 4. During this final phase, which lasted 21 days ( 7 cycles), the groups that had formerly consumed the LS diet were returned to the $C$ diet.

\section{Data Analysis}

For each animal, daily fluid consumption was converted to milliliters of ethanol solution or water per kilogram body weight. For the purpose of analysis, the study was treated as consisting of two between-subjects factors (diet, i.e., LS/C, and ethanol concentration, j.e., 3\%, 6\%, and $12 \%$ ) and one within-subject factor (phase). Since we were interested in the change in consumption beween the adjacent phases, the phase factor was partitioned into three individual contrasts, the first contrast represent- ing the change from Phase 1 to Phase 2, the second contrast representing the change from Phase 2 to Phase 3, and the third contrast comparing Phase 3 and 4 . Thus, this analysis is a three-way ANOVA, with the within-subject factor, phase, being partitioned into three individual contrasts. However, for ease of interpretation, the analysis can be viewed as though separate two-way ANOVAs had been performed on each of the three contrasts. For example, a three-way interaction of diet $x$ concentration $\times$ Contrast 1 for ethanol consumption can be interpreted as a two-way interaction of diet $\times$ concentration for the difference in ethanol consumption between Phase 1 and Phase 2 .

The criterion for significance for the F statistic was set at the .01 level and post hoc analysis within each of the three ethanol concentrations was done using paired $t$ tests (.05 level).

\section{RESULTS}

Significant changes in choice-day ethanol consumption were found only between Phases 2 and $3[F(1,27)=10.08$, $\mathrm{p}<.004]$ and between Phases 3 and $4[\mathrm{~F}(1,27)=14.54$, $\mathrm{p}<.0007]$. Furthermore, these changes depended on the particular diet (LS vs. C) consumed [Phases 2-3, F(1,27) $=9.75, \mathrm{p}<.004$; Phases 3-4, $\mathrm{F}(1,27)=8.49, \mathrm{p}<.007]$. There were no significant differences in choice-day ethanol consumption among the three dose groups, nor were there any significant diet $\times$ dose interactions.

Significant changes in choice-day water consumption $\propto$ curred between Phases 1 and $2[\mathrm{~F}(1,27)=17.64$, $\mathrm{p}<.003]$, between Phases 2 and $3[\mathrm{~F}(1,27)=61.79$, $\mathrm{p}<.0001]$, and between Phases 3 and $4[\mathrm{~F}(1,27)=67.04$, $p<.0001]$. These changes depended on the particular diet consumed only between Phases 2 and $3[F(1,27)=36.41$, $\mathrm{p}<.0001]$ and between Phases 3 and $4[\mathrm{~F}(1,27)=24.69$, $\mathrm{p}<.0001]$. Significant differences among the three dose groups occurred between Phases 1 and $2[\mathrm{~F}(2,27)=7.95$, $\mathrm{p}<.002]$. There were no other significant dose or diet $x$ dose interactions.

\section{Effects of Low-Sodium Diet/Furosemide on Choice-Day Intake of Ethanol and Water}

Figure 1 gives the mean ethanol and water intake on choice days for the LS groups across the four phases of the experiment. For both the $3 \%$ and $6 \%$ groups, the LS diet alone did not produce any significant change in the intake of either ethanol or water (Phase 2). However, the addition of the four furosemide injections to the LS diet at the beginning of Phase 3 produced significant decreases in ethanol intake $[3 \%, \mathrm{t}(5)=2.36, \mathrm{p}<.04 ; 6 \%, \mathrm{t}(5)$ $=3.19, \mathrm{p}<.03]$ and significant increases in water intake $[3 \%, \mathrm{t}(5)=3.53, \mathrm{p}<.02 ; 6 \%, \mathrm{t}(5)=15.4$, $\mathrm{p}<.0001]$. These effects lasted far beyond the last furosemide injection. For example, mean ethanol consumption for the 3\% LS group immediately after the last furosemide injection (Day 12) and on the final choice day (Day 30) was 64.1 and $61.4 \mathrm{ml} / \mathrm{kg}$, respectively. Furthermore, when the animals were returned to the control diet in Phase 4, there was a return of their respective intake of both ethanol $[3 \%, \mathrm{t}(5)=3.75, \mathrm{p}<.01 ; 6 \%, \mathrm{t}(5)=$ 2.37 , nonsignificant tendency $]$ and water $[3 \%, \mathrm{t}(5)=3.28$, $p<.02 ; 6 \%, t(5)=4.30, p<.01]$. Since animals in the $12 \%$ group drank very little ethanol, it is not surprising that neither the LS diet nor the combination of diet and 


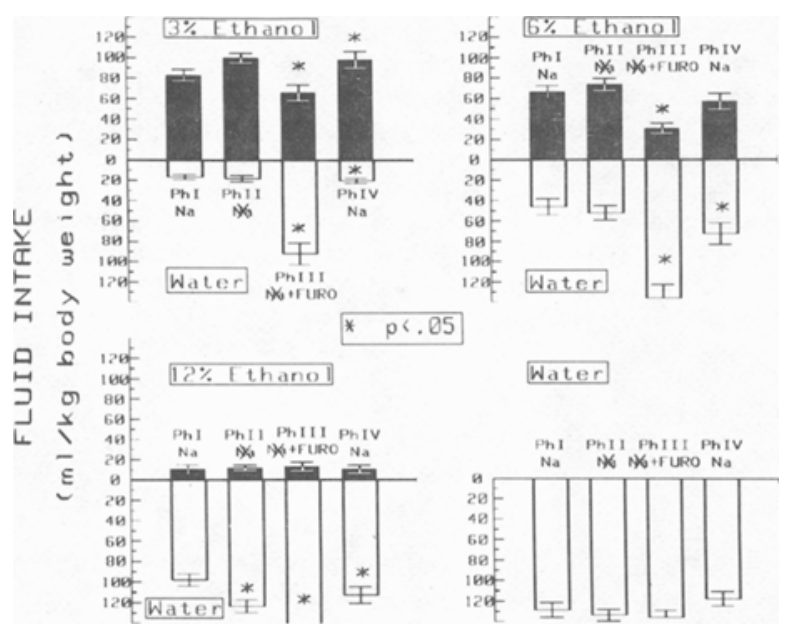

Figure 1. Mean ethanol (black histograms) and water intake (white histograms) on choice days for the LS groups drinking ethanol (3\%, $6 \%$, or $12 \%$ ) or water across the four phases of the experiment. Asterisks $(*)$ indicate the phases in which consumption was significantly different ( $p<.05$ or better) from the immediately preceding phase. Bars repesent \pm the standard error of the man. $\mathrm{Na}$ indicates the availability of control diet; 1 indicates availability of low sodium diet; Furo indicates four injections of furosemide $(60 \mathrm{mg} / \mathrm{kg})$ at the beginning of Phase 3 .

furosemide had any effect on intake. On the other hand, water intake was increased both by the diet itself $[\mathrm{t}(5)=$ $3.76, \mathrm{p}<.02]$ and by the addition of furosemide $[\mathrm{t}(5)=$ $3.72, \mathrm{p}<.02$ ], returning to control levels when the control diet was reinstated $[\mathrm{t}(5)=6.41, \mathrm{p}<.003]$. The water group, which was never offered ethanol, showed no significant changes in intake across any of the phases of the experiment, even though acute (24-h) increases in intake subsequent to the furosemide-induced diuresis were sometimes noted (e.g., after the last furosemide injection on Day 12).

Taken together, these findings indicate that the LS diet is not sufficient in itself to reduce ethanol consumption and that the added diuresis-natriuresis must occur to significantly decrease ethanol intake. Furthermore, the changes in ethanol and water intake are not irreversible, but are tied strictly to the changes induced by the dietfurosemide combination. It is possible that the decrease in ethanol intake in Phase 3 was secondary and compensatory to the increase in the water intake that also occurred in this phase. However, the fact that the brief administration of furosemide in combination with the LS diet in this phase was not sufficient to produce a sustained increase in the water intake of the water group suggests that this manipulation had its primary effect on ethanol intake.

\section{Effects of Control Diet/Furosemide on Ethanol and Water Intake}

Figure 2 gives the results for the animals that were maintained on the control diet throughout and also received four injections of furosemide in Phase 3. Both the 3\% and $6 \%$ groups showed no statistically significant change in ethanol intake between each of the four phases, although there was a tendency for intake to increase in the $3 \%$ group. The $12 \%$ group did show a significant decrease in intake during Phase $3[\mathrm{t}(5)=5.08, \mathrm{p}<.004]$. However, since this group had access to the control diet and could therefore restore any diuretic-induced fluid and electrolyte loss, this decrease is not likely to be related to the furosemide treatment itself. For the water group, no significant change in intake was noted, and even though significant changes in water consumption in the $3 \%[\mathrm{t}(4)=4.93, \mathrm{p}<.008]$, $6 \%$ [Phase 2, $\mathrm{t}(5)=2.9, \mathrm{p}<.03 ;$ Phase $4, \mathrm{t}(5)=3.4$, $\mathrm{p}<.02]$, and $12 \%[\mathrm{t}(5)=3.00, \mathrm{p}<.03]$ groups did occur, they did not appear to be related systematically to any change in treatment.

Taken together, these findings show that neither a chronic exposure to ethanol nor a brief treatment with the diuretic per se are sufficient to reduce ethanol intake, and indicate that the combined manipulation of the LS diet and diuretic are necessary to produce the observed changes in drug intake.

\section{Effects of Low Sodium and Control Diet/Furosemide on Forced Ethanol Intake}

On the 2 days intervening between choice days, each group was offered only its respective solution of ethanol. Significant changes in forced ethanol intake were not observed across the different phases [Phases $1-2, F(1,27)=$ 0.06; Phases 2-3, $F(1,27)=3.96$; Phases $3-4, F(1,27)=$ 2.03]; there were no significant effects of diet [Phases 1$2, \mathrm{~F}(1,27)=0.13$; Phases 2-3, $\mathrm{F}(1,27)=0.51$; Phases 3$4, F(1,27)=1.21]$, no significant effects among the three dose groups [Phases 1-2, $\mathrm{F}(2,27)=0.98$; Phases 2-3, $\mathrm{F}(2,27)=2.43$; Phases $3-4, F(2,27)=1.34]$, and no sig-

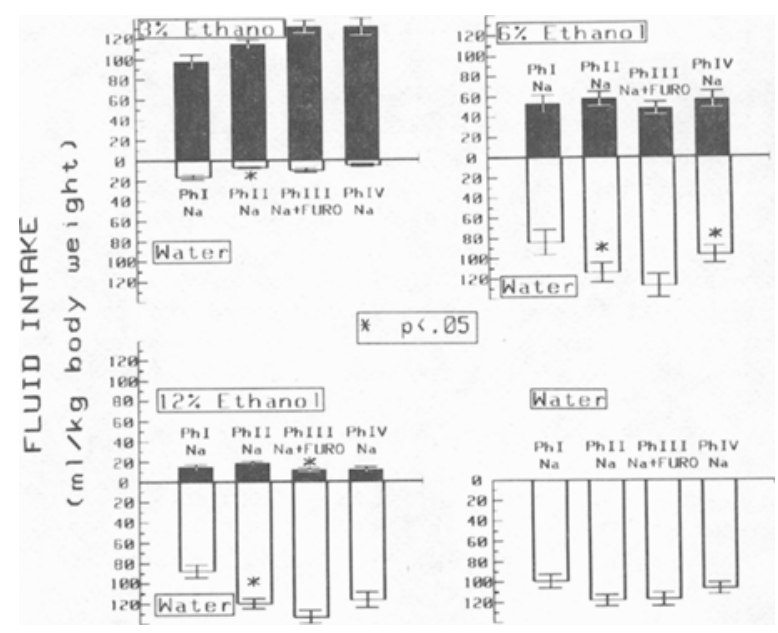

Figure 2. Mean ethanol (black histograms) and water intake (white histograms) on the choice days for the control groups drinking ethanol $(3 \%, 6 \%$, or $12 \%)$ or water across the four phases of the experiment. Asterisks (*) indicate the phases in which consumption was significantly different $(p<.05$ or better) from the immediately preceding phase. Bars represent \pm the standard error of the mean. $\mathrm{Na}$ indicates the control diet; Furo indicates four injections of furosemide $(60 \mathrm{mg} / \mathrm{kg})$ at the beginning of Phase 3. 
nificant diet $x$ dose interactions, except between Phases 3 and $4[F(2,27)=5.12, p<.01]$. The groups offered only water showed no significant changes in intake across the different phases [Phases 1-2, $F(1,10)=0.11$; Phases 2$3, F(1,10)=0.96$; Phases $3-4, F(1,10)=0.03]$, and no significant effects of diet [Phases 1-2, $F(1,10)=1.61$; Phases 2-3, $\mathrm{F}(1,10)=0.69$; Phases $3-4, \mathrm{~F}(1,10)=0.49$ ]

Figure $3 \mathrm{~A}$ gives the mean intake for the groups on the $\mathrm{C}$ diet and shows that little change in intake occurred over the four phases of the experiment. Figure 3B shows that neither the imposition of the LS diet (Phase 2) nor the brief addition of furosemide injections to that diet (Phase 3) produced any significant change in forced ethanol intake. The effect that the LS diet/furosemide manipulation has in reducing ethanol intake on choice days is not evident when ethanol is the only liquid available to maintain fluid balance. This suggests that the integrity of the animal's fluid balance outweighs the otherwise countervailing influence of this manipulation. Although water intake did increase on choice days during the LS diet/furosemide phase (Phase 3, Figure 1), no similar increase in water intake was evident during forced days in the group drinking water. This finding corroborates the earlier suggestion that the effect of the manipulation on intake during the choice days is primarily on ethanol intake and that the increase in water drinking is compensatory to this.

\section{DISCUSSION}

The main finding of the present study is that dietary salt restriction, when combined with a brief series of injections of a diuretic-natriuretic agent, led to a significant

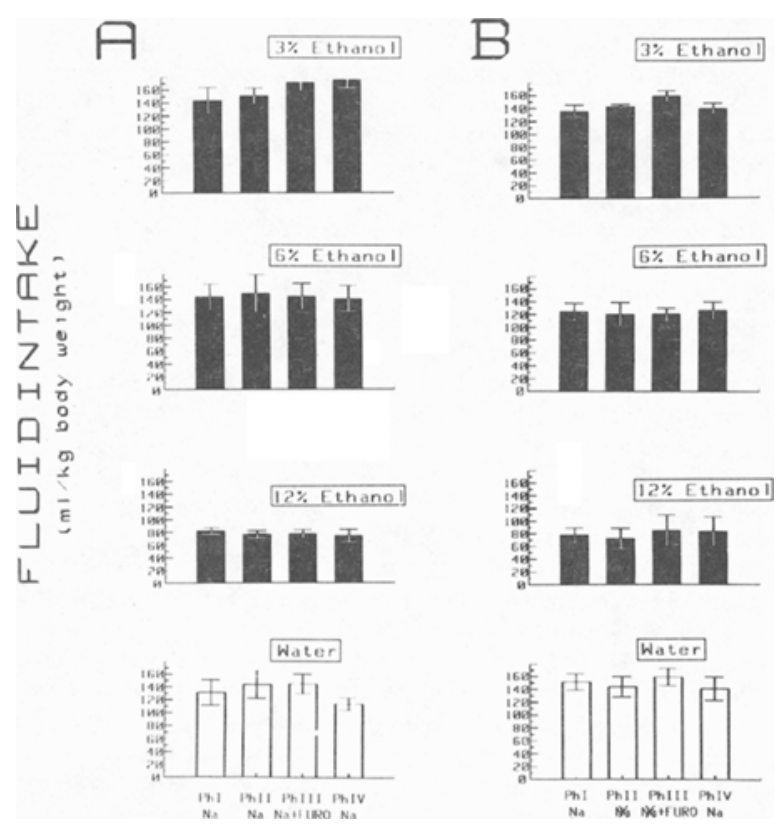

Figure 3.Mean forced-day consumption of ethanol $(3 \%, 6 \%, 12 \%)$ or water for the LS groups (Panel B) or the control diet groups (Panel A) across the four phases of the experiment. All conventions are as per Figure 1. decrease in the voluntary intake of ethanol solutions whose concentrations $(3 \%, 6 \%)$ are known to be acceptable to rats (Richter \& Campbell, 1940). Neither salt restriction alone nor the administration of the diuretic-natriuretic agent by itself were able to bring about this suppressive effect on ethanol intake.

Although the mechanism by which this occurs is not clear, certain possibilities can be ruled out. The first centers around the notion that the present manipulation might lead to a change in the sensitivity of the taste receptors and thus decrease intake by rendering ethanol less palatable. Two findings argue against this: (1) Pfaffmann and Bare (1950) and Pfaffmann (1957) found no change in the physiological response of the taste buds of animals who were deprived of sodium; and (2) Yensen (1958) found that sodium-deficient subjects showed enhanced sensitivity to salty tastes but no change in their sensitivity to sweet, sour, or bitter tastes. Taken together, these studies suggest that the decreased ethanol intake in the present experiment is not likely to be due to a change in palatablity of the ethanol solutions.

Another possibility is that the decreased intake of ethanol may reflect the animal's attempt to retain sodium in the face of any loss induced by either the LS diet or the diuretic. Such an explanation assumes that ethanol intake enhances sodium loss through the diuresis induced by ethanol's inhibitory effect on vasopressin, and that decreasing ethanol intake would curtail such a loss. However, Sargent, Simpson, and Beard $(1974,1980,1981)$ have shown, for both acute and chronic administration, that ethanol leads to a net retention and not loss of sodium through the urine. It is therefore unlikely that the change in ethanol intake is secondary to the animal's attempt to regulate sodium.

Although ethanol intake decreased on choice days when water was also available to drink, the same animals did not decrease their intake on the 2 forced days when only ethanol was present. This suggests that the need to maintain fluid balance retains its priority and remains unaffected by factors that would otherwise reduce ethanol consumption. Whatever their nature, these factors apparently do not operate outside the boundaries set by the systems that regulate fluid balance.

Although the salt-restricted animals were maintained for 39 days on the LS diet (Phase 2), it was only after the addition of the furosemide injections during the initial part of Phase 3 that a significant and sustained decrease in ethanol intake occurred. Furthermore, furosemide alone administered to the animals on the control diet did not alter ethanol intake. Taken together, this argues for the possibility that the effect on ethanol intake may be more related to some chronic consequence of the combined LS/furosemide treatment than to an acute disruption of fluid balance that occurs as a result of the furosemide-induced diuresis/natriuresis. Although no direct electrolyte or hormonal assays were taken in this study, it is well known that the diet and furosemide manipulation can lead to a number of related effects, including a sodium loss (e.g., Jalowiec, 1974; Schulkin, 1978; Wolf, McGovern, \& Dicara, 1974), 
hypovolemia (e.g., Jalowiec, 1974) and an increased activity in the renin-angiotensin-aldosterone axis (Jalowiec, 1974; Leenen, 1981; Spät, Tarjan, \& Toth, 1979). The extent to which any of these consequences of the diet-diuretic manipulation play a role in reducing ethanol intake remains to be determined.

Without specific inducing conditions, such as scheduleinduced polydipsia or severe food deprivation, rats will not usually consume sufficient amounts of ethanol to produce overt intoxication. In the present experiment, in which the animals were fed ad lib, no special attempt was made to encourage prodigious consumption and only moderate amounts of ethanol were consumed on the choice days of Phases 1,2 , and 4 (approximately $2.5 \mathrm{~g} / \mathrm{kg} /$ day for the $3 \%$ group and $3.5 \mathrm{~g} / \mathrm{kg} /$ day for the $6 \%$ group). However, it is known that animals undergoing a diuresisnatriuresis without the concurrent availability of sufficient sodium in the diet, as was the case in Phase 3, have a reduced extracellular fluid volume and therefore a reduced volume of distribution (Jalowiec, 1974). This will result despite the continuous availability of liquid because the lack of sodium precludes the permanent restoration of fluid volume (Stricker \& Wolf, 1969). Under such conditions, the failure to reduce ethanol intake may result in severe intoxication, a state which can be aversive (Grupp, 1981; Grupp \& Stewart, 1983). The reduced ethanol drinking in Phase 3, therefore, may simply reflect the animal's attempt to regulate its intake so as to prevent any untoward effects of too much drug.

In summary, the present investigation indicates that salt restriction in combination with the salt-losing diuretic, furosemide, is an effective means of reducing ethanol consumption. Future research will be directed towards testing this manipulation under conditions of higher ethanol intake levels and toward investigating how the various physiological and pharmacological consequences of the manipulation contribute to its suppressive effect on ethanol intake.

\section{REFERENCES}

AsHLEY, M. I., \& RANKIN, J. G. (1979). Alcohol consumption and hypertension: The evidence from hazardous drinking and alcoholic populations. Australian and New Zealand Journal of Medicine, 9, 201-206.

DAHL, L. K. (1977). Salt intake in hypertension. In J. Genest, E. Koiw, \& O. Kuchel (Eds.), Hypertension (pp. 548-559). New York: McGraw-Hill.

DENTON, D. A. (1967). Salt appetite. In Handbook of experimental physiology. The alimentary canal: Control of food and water intake (Vol. 2, pp. 443-459). Washington, DC: Physiological Society.

EPSTEIN, M., SANCHO, J., \& HABER, E. (1979). Renin-aldosterone responsiveness in decompensated cirrhosis. In M. Epstein (Ed.), The kidney in liver disease (pp. 225-237). New York: Elsevier.

GRUPP, L. A. (1981). Ethanol as the negative reinforcer in an active avoidance paradigm. Progress in neuro-psychopharmacology, 5, 241-244.

Grupp, L. A., Elias, J., Perlanski, E., \& Stewart, R. B. (in press). Modification of ethanol-induced motor impairment by diet diuretic, mineralocorricoid or prostaglandin synthetase inhibitor. Psychopharmacology.

Grupp, L. A., Perlanski, E., \& Stewart, R. B. (1984). Dietary salt and DOCA-salt treatments modify ethanol self-selection in rats. $B e-$ havioral \& Neural Biology, 40, 239-250.
GrupP, L. A., \& Stewart, R. B. (1983). Active and passive avoidance behaviour in rats produced by intravenous infusions of ethanol. Psychopharmacology, 79, 318-321.

IIDA, S. (1957). Experimental studies on the craving for alcohol. I. Alcoholic drive in mice following administration of saline. Japanese Journal of Pharmacology, 6, 87-93.

JALOWIEC, J. E. (1974). Sodium appetite elicited by furosemide: Effects of differential dietary maintenance. Behavioral Biology, 10, 313-327.

LEENEN, F. H. H. (1981). Diuretic and cardiovascular effects of furosemide in rats. Canadian Journal of Physiology and Pharmacology, 59, 1002-1007.

LiNkoLA, J. (1982). Strain differences in water and electrolyre metabolism between alcohol preferring $(A A)$ and alcohol avoiding (ANA) rats (pp. 1-96). Helsinki: Minerva Foundation for Medical Research.

Linkola, J., Fyhrouist, F., \& Forsander, O. (1977). Effects of ethanol on urinary arginine vasopressin excretion in two rat strains selected for their different ethanol preferences. Acta Physiologica Scandinavica, 101, 126-128.

MAthews, J. D. (1979). Alcohol and hypertension. Australian and New Zealand Journal of Medicine, 9, 124-128.

PfaffmanN, C. (1957). Taste mechanisms in preference behavior. American Journal of Clinical Nutrition, 5, 142-147.

PfaffmanN, C., \& BARE, J. K. (1950). Gustatory nerve discharges in normal and adrenalectomized rats. Journal of Comparative Physiological Psychology, 43, 320-324.

RICHTER, C. P. (1942-1943). Total self regulatory functions in animals and human beings. The Harvey Lectures, 38, 63-103.

Richter, C. P., \& CAMPBELl, K. H. (1940). Alcohol taste thresholds and concentrations of solution preferred by rats. Science, 91, 507-508.

RuBIN, E. (1982). Alcohol and the heart: Theoretical considerations. Federation Proceedings, 41, 2460-2464.

Sargent, W. Q., Simpson, J. R., \& BeARD, J. D. (1974). Effect of acute and chronic alcohol administration on renal hemodynamics and monovalent ion excretion. Joumal of Pharmacology and Experimen tal Therapeutics, 188, 461-471.

Sargent, W. Q., Simpson, J. R., \& Beard, J. D. (1980). Twentyfour-hour fluid intake and renal handling of electrolytes after various doses of ethanol. Alcoholism: Clinical and Experimental Research, 4, 74-88.

Sargent, W. Q., Simpson, J. R., \& Beard, J. D. (1981). Water metabolism and monovalent in excretion during one day of multiple ethanol doses. Substance and Alcohol Actions/Misuse, 2, 301-310.

SCHULKIN, J. (1978). Mineralocorticoids, dietary conditions and sodium appetite. Behavioral Biology, 23, 197-205.

SMITH, J. J. (1950). The treatment of acute alcoholic state with ACTH and adrenocortical hormones. Quarterly Journal of Studies on Alcoholism, 11, 190-198.

SPÄt, A., Taruan, E., \& Toth, G. (1979). Hyperaldosteronism in the sodium-depleted rat: Mode of aldosterone-stimulating action of furosemide. Journal of Endocrinology, 82, 7-15.

STRICKER, E. M., \& WOLF, G. (1969). Behavioral control of intravascular fluid volume: Thirst and sodium appetite. Annals of the New York Academy of Science, 157, 553-568.

Wilkins, L., \& RichTER, C. P. (1940). A great craving for salt by a child with corticoadrenal insufficiency. Journal of the American Medical Association, 114, 866-868.

Wolf, G., McGovern, J. F., \& Dicara, L. V. (1974). Sodium appetite: Some conceptual and methodological aspects of a model drive system. Behavioral Biology, 10, 27-42.

Woodbury, D. M., Timiras, P. S., \& Vernadakis, A. (1957). Influence of adrenocortical steriods on brain function and metabolism. In $\mathrm{H}$. Hoaglund (Ed.), Hormones, brain function and behaviour. New York: Academic Press.

YENSEN, R. (1958). Influence of salt deficiency on taste sensitivity in human subjects: Nature, 181, 1472-1474.

(Manuscript received June 16, 1983; revision accepted for publication June 25,1984 .) 